Emil Angehrn

\title{
ZivilgeSEllschaft UND STAAT. ANMERKUNGEN ZU EINER DISKUSSION
}

In: Politisches Denken. Jahrbuch 1992, Stuttgart: Metzler 1993, S. 145-158

1.

Die Schwierigkeit, sich in der seit einigen Jahren in Umlauf gekommenen Diskussion über Zivilgesellschaft Klarheit zu verschaffen, ist eine mehrfache. Zum einen hat sie mit Herkunft und Bezugsfeld der Diskussion zu tun. Die in den beiden letzten Jahrzehnten in osteuropäischen Staaten geführte Diskussion bewegt sich im Horizont einer Sichtung von Oppositionsformen und demokratischen Alternativen zum herrschenden Staatsapparat und verweist mit dem Titel der civil society auf das, was fehlt: auf nicht vom Staat integrierte Formen gesellschaftlicher Tätigkeit und Selbstorganisation. Viele haben den 1989 kulminierenden Prozeß als Wiedergewinnung der Zivilgesellschaft beschrieben ${ }^{1}$. Angesprochen ist darin ein ganzes Syndrom von praktischen Leitmotiven und institutionellen Vorstellungen, die nicht ohne weiteres auf einen Nenner zu bringen sind. Zum Teil verwandte Motive bestimmen die westliche Diskussion, die ihrerseits ein Ungenügen an der realen Verfaßtheit des Politischen zum Ausdruck bringt - eine Gemeinsamkeit, die allerdings ihre Grenzen hat: Manches, was dort zum vitalen Anliegen gehört(e) - der Übergang zum pluralistischen Mehrparteiensystem und zur liberalen Marktwirtschaft -, ist hier Teil der als defizient kritisierten Realität. Schon dies läßt fraglich werden, ob die grenzüberschreitende Diskussion einen einheitlichen Fokus habe. In der deutschsprachigen Diskussion kommt die eigenartige Doppelterminologie hinzu: "Zivilgesellschaft" oder "civil society" kommen als Alternativbegriffe neben "bürgerliche Gesellschaft" zu stehen, womit sich irgendwie die Unterstellung verbindet, daß der letzte Begriff für das verhandelte Anliegen zu eng oder zu einseitig sei; "bürgerliche Gesellschaft" bleibt gewissermaßen zu stark durch die Hegelsche Prägung und die Marxsche Diskreditierung besetzt, um im Diskurs frei verfügbar zu sein. Zivilgesellschaft hat mit dem Bürgerlichen zu tun, wobei aber als semantische Anschlußstellen teils eher Kontexte wie "ziviler Ungehorsam" und "Bürger(rechts)bewegungen", "Bürgerinitiativen" etc. fungieren.

Die schon öfters monierte theoretische Ungeklärtheit des Begriffs in der gegenwärtigen Diskussion $^{2}$ affiziert auch die Verhältnisbestimmung von Zivilgesellschaft und Staat und

\footnotetext{
${ }^{1}$ Ash 1990, 224; U.K.Preuß, in: Th.Blanke/R.Erd (Hg.), DDR. Ein Staat vergeht, Frankfurt 1990, 84. - Vgl. Keane 1988, Michalski 1988

${ }^{2}$ Vgl. Honneth 1992, Keane 1988, 6ff.
} 
verweist dabei zum ersten auf die Tatsache, daß hier mit einem Gegensatz operiert wird, der etymologisch und sachlich so zunächst gar nicht gegeben ist. Vom Eingangssatz der aristotelischen Politik bis zu Kants Metaphysik der Sitten ( $\S 45 f$.) und teils darüber hinaus gelten Staat und bürgerliche Gesellschaft als dasselbe. Dabei ist die Gleichsetzung von polis und koinonia politike, civitas und societas civilis nicht so zu verstehen, daß wir hier einfach über zwei austauschbare Namen für dasselbe verfügten. Vielmehr hat sie genauen definitorischen Sinn: Der Staat wird aus den verschiedenen menschlichen Gemeinschaften als die politische/zivile hervorgehoben und dadurch von anderen Formen der Vereinigung, allen voran der häuslichen/ökonomischen abgehoben ${ }^{3}$. Die begriffsgeschichtliche Zäsur findet bekanntlich bei Hegel statt, der die Differenz von Staat und bürgerlicher Gesellschaft ausdrücklich gegen deren Verwechslung (etwa in naturrechtlichen Vertragstheorien) unterstreicht ${ }^{4}$ und zugleich darauf verweist, daß es hier nicht um eine rein begriffliche Distinktion, sondern um den Nachvollzug einer realen Differenzierung geht, die als solche ein Produkt der Moderne ist ${ }^{5}$. Modern kann man sie zum einen im Blick auf die von Luhmann betonte funktionale Differenzierung der Gesellschaft nennen; zum anderen mit Bezug auf das von Hegel unterstrichene Recht der subjektiven Freiheit, das seinen institutionellen Rahmen vorab in der bürgerlichen Gesellschaft findet.

Nun ist nicht Hegels Unterscheidung als solche für die heutige Diskussion bestimmend, ebenso wenig wie andere traditionelle Begriffsprägungen. Honneth nennt (in Weiterführung einer Unterscheidung von Ch.Taylor) drei Kristallisationspunkte: erstens den (etwa bei Locke beschriebenen) vorpolitischen Zusammenschluß der Bürger in den Medien des Wirtschaftens und der öffentlichen Meinungsbildung, zweitens die (etwa bei Montesquieu und Toqueville präsente) Ebene der rechtlich-politisch verfaßten Assoziationen und Körperschaften der öffentlichen Selbstverwaltung, welche Gegeninstanzen zu Staat und Regierung bilden und diese kontrollieren, drittens die von Gramsci als "società civile" geschilderte Sphäre der öffentlichen Meinungsbildung, die zum ökonomischen wie politischen Funktionsbereich gleichermaßen Abstand hält, aber als Konsens- und Wertbildung auf beide Einfluß nimmt. Es kann hier nicht darum gehen, das Spektrum der Begriffsverwendungen auszuleuchten oder den adäquaten Begriff zu definieren; dazu wäre eine sowohl systematisch wie geschichtlich - realhistorisch und ideengeschichtlich - differenzierte Analyse erforderlich. Statt dessen soll allein versucht werden, einige bestimmende Motive auszumachen, die in gegenwärtigen Dikussionen mit dem Begriff der Zivilgesellschaft ins Spiel gebracht werden. Dazu empfiehlt es sich, nicht vom Begriff in

\footnotetext{
${ }^{3}$ Noch bei Christian Thomasius (1725) heißt es: "Die Gesellschaft der Menschen an sich selbst ist entweder bürgerlich oder häuslich" (Riedel 1969, 144); vgl. Riedel 1975

${ }^{4}$ Rechtsphil. §33 Zusatz, §258 Anm.

${ }^{5}$ Rechtsphil. §182 Zusatz
} 
seiner weitesten Bedeutung auszugehen, sondern ihn mit einer doppelten Spezifizierung aufzunehmen. Zum einen ist offenkundig, daß in gegenwärtigen Debatten unter dem Titel der Zivilgesellschaft etwas interessiert, was dem Politischen oder Staatlichen nicht einfach als das Andere gegenübersteht. Nicht das System der Bedürfnisse als solches und nicht Bildung und Kultur als solche stehen im Blick: Zivilgesellschaft ist ein Thema politischer Reflexion und beschäftigt sich (u.a.) mit jenen Bereichen, soweit sie in einem bestimmten Verhältnis zum Politischen stehen (auf es bestimmte Auswirkungen haben, für es bestimmte Funktionen erfüllen), als selber in einem weiten Sinn politische Größen. Zum andern liegt darin, daß der Begriff, als politischer, durch seinen Gegensatz zum Staat definiert ist: Er ist insofern negativ bestimmt, teils Komplementärbereich zum Staat, teils Sammelbegriff für Vorstellungen und Motive, mit denen auf ein Ungenügen des Staats, auf ein Unbehagen am Staat reagiert wird. Bürgerinitiativen und ziviler Ungehorsam verstehen sich als solche Korrektive, welche Entscheidungen der politischen Instanzen beeinflussen, konstitutive Defizite des Systems kompensieren, funktionale und normative Lücken füllen. Zwar erschöpft sich der zivile Impuls nicht in der Antithese; ebenso kann er Ergänzungs- oder Begründungsfunktionen für Politik übernehmen. Gleichwohl legt es sich nahe, eine Verständigung über Zivilgesellschaft zunächst an der kritischen Stoßrichtung des Begriffs festzumachen; dazu sind als erstes Aspekte der Kritik am Staat zu präzisieren (2.). Danach sind Funktionen der Zivilgesellschaft (3.), ihre Interferenz mit der Institutionalisierung des Politischen (4.) sowie Grenzen des zivilgesellschaftlichen Ideals (5.) aufzuzeigen.

2.

a) Die Kritik am Staat aus der Sicht der Gesellschaft ist so alt wie die Formulierung ihrer Differenz: Marx' Abrechnung mit Hegels Rechtsphilosophie ist Kritik am Staat als Kritik an der Differenz. Differenz heißt hier Entfremdung: Kritisiert wird der Staat als eine vom Handeln der Subjekte abgelöste, verselbständigte Sphäre des Sozialen, welche den Individuen und jeder lebendigen Gemeinschaft als fremde Macht gegenübertritt und über sie herrscht. Die Gesellschaft soll sich die entäußerten Potenzen der Selbstregulierung wieder aneignen; die Utopie einer unmittelbaren Sozialität, an welche die spätere Foderung einer Aufhebung des Staats anschließt, will die nicht-entfremdete Arbeit und lebendige Gemeinschaft in direktester Weise verknüpfen. Es ist ein Ideal, dessen sozialphilosophische Fragwürdigkeit auf der Hand liegt: Sein strenger Anti-Institutionalismus läßt fraglich werden, ob in ihm eine Alternative (und nicht nur ein Komplementärmoment) zu einem wie immer vereinseitigten Staat zu suchen ist. Gleichwohl bildet die Stoßrichtung der Kritik ein bedeutsames Element einer Gegenkonzeption zur bestehenden Form des Politischen. 
b) In gewisser Affinität dazu steht die Kritik am Staat als identitärem Gebilde. Das Stichwort des Identitären, das Castoriadis zu einem Angelpunkt seiner Theorie des gesellschaftlich Imaginären als des Ursprungs historischer Kreativität macht, steht für die fixierende Vergegenständlichung, die sich dem schöpferischen Impuls widersetzt; es wird von Rödel, Frankenberg und Dubiel zur Charakterisierung einer verengenden Institutionalisierung des Politischen übernommen. In ihm verbindet sich - wie in anderen negativ konnotierten Verwendungen des Identitätsbegriffs (etwa in der Rede vom Identitätszwang) - die falsche Vergegenständlichung mit der falschen Vereinheitlichung: die starre Verfestigung gegen die "alltägliche Neugründung" des Politischen ${ }^{6}$ mit der Homogenisierung und Singularisierung kollektiver Identität in einem Staat, einer Nation. Im weiteren Rahmen wird unter dem Stichwort der Mangel an Pluralität und Liberalität im politischen System problematisiert.

c) Gleichsam gegenläufig dazu artikuliert sich das Unbehagen an der modernen Dezentrierung des Politischen, wie sie nach Luhmann aus der funktionalen Differenzierung der modernen Gesellschaft resultiert: Politik ist nicht mehr das Ganze und Höchste, sondern ein Sonderbereich neben anderen und damit erneut, wenn auch in anderem Sinn, von der lebendigen Sozialität abgespalten und den einzelnen fremd geworden. Sie ist intern durch den Machtkampf wechselnder Mehrheiten, nach außen durch die Herstellung bindender Entscheidungen definiert, nicht mehr Gesamtrahmen einer kollektiven Identitätsbildung oder normativen Selbstverständigung. Das Unbehagen, das hier zum Ausdruck kommt, ist nicht mehr unmittelbarer Protest gegen den Staat, sondern ein Unbehagen an der Verfaßtheit der Gesamtgesellschaft, deren Ausdifferenzierung Orientierungsverluste und Dysfunktionalitäten mit sich bringt (etwa für die Lösung gesamtgesellschaftlicher, z.B. ökologischer Probleme). Man mag in der Auflehnung dagegen einen Protest gegen die funktionale Differenzierung als solche ${ }^{7}$, ja gegen die Moderne sehen - auch wenn kein Zufall ist, daß sich dieser in bevorzugter Weise am Politischen und seiner Selbstblockierung festmacht.

d) Strittig ist im weiteren die Aufgabenteilung zwischen Gesellschaft und Staat, und Anstoß erregt die unzulängliche Leistungsfähigkeit des letzteren. Die Stellungnahmen zum ersten Punkt divergieren je nach politischer Position; wo die einen mangelhafte Steuerung beklagen, kritisieren andere den Interventionismus. Unkontrovers ist die Grenze staatlicher Effizienz, die mangelhafte Realisierung sozial- und wohlfahrtsstaatlicher Ziele; sie läßt Selbsthilfegruppen und -organisationen entstehen, die teils Funktionsdefizite der Verwaltung kompensieren, teils in gewollter Distanz zu staatlichen Instanzen und Monopolen tätig sind.

\footnotetext{
${ }^{6}$ Rödel u.a. 1989,71

${ }^{7}$ Niklas Luhmann, Ökologische Kommunikation, Opladen 1986, 234
} 
e) Schließlich wird das Demokratiedefizit herrschender Systeme angeklagt, und zwar nicht nur der Scheindemokratien des bürokratischen Sozialismus, sondern der parlamentarischen, repräsentativen Demokratien des Westens. Die Intransparenz der Machtkanäle und Interessenverflechtungen, die Schwerfälligkeit und Vermitteltheit persönlicher Einflußnahme, die Entstehung von Eigengesetzlichkeiten des Systems, das Übergewicht von Wirtschaft und Verwaltung usw. lassen den Ruf nach Demokratisierung von Staat und Gesellschaft aufkommen. In gewissem Sinn ist damit der zentrale Bezugspunkt für die Problematisierung des Staats und indirekt auch für die Thematisierung der Zivilgesellschaft benannt. Das Problem der Demokratie, wie es sich in zugespitzter Form bei Rousseau zeigt, besteht darin, Freiheit und Gleichheit so zu vereinbaren, daß der einzelne in der Gemeinschaft nichts von seiner Freiheit aufgibt. Die Schwierigkeit kollektiver Selbstbestimmung offenbart sich in der Strenge der Leitvorstellungen, der volonté générale und der damit einhergehenden Moralisierung der Bürger. Nicht nur wird ein kollektives Ethos als Grundlage der Gesellschaft, sondern eine reale Übereinstimmung postuliert, die nicht nur die Rahmenbedingungen des Zusammenlebens, sondern den jeweiligen Mehrheitswillen betrifft: Die paradoxe Auflösung des MehrheitMinderheit-Problems, wonach die Minderheit nicht ihr Überstimmtwerden, sondern ihre Selbsttäuschung mit Bezug auf das wahre Allgemeine (das auch sie "an sich" gewollt haben soll) akzeptieren muß, zeigt die Unhaltbarkeit der Konstruktion. Die Tyrannei der Mehrheit wird durch den Tugendzwang für den einzelnen nur scheinbar gemildert. Der Ausweg ist zunächst die Ebenendifferenzierung, die das zustimmungspflichtige Allgemeine nur auf der Ebene der Institution bzw. des Verfahrens ansiedelt und damit den Raum für die Eigeninteressen der einzelnen freigibt. Das generelle Erfordernis lautet, die Vermittlung von Allgemeinheit und Besonderheit zu leisten, die individuelle Selbstbestimmung wie die Durchsetzbarkeit eines Allgemeinen zu ermöglichen. Das Spannungsverhältnis der Erfordernisse legt es nahe, die Lösung nicht in einer kompakten Institution zu suchen; die institutionelle Gewährleistung jener Vermittlung weist zunächst auf die spezifische Ausgestaltung des politischen Systems (Gewaltenteilung, Partizipationsmechanismen).

Im weiteren aber zeigt sich, daß hier Desiderate artikuliert werden, die nicht im Binnenbereich des Politischen allein zu erfüllen sind. Sofern man hier das Konzept einer Zivilgesellschaft ins Spiel bringt (und sich nicht mit systemimmanten Korrektiven, etwa der erhöhten Bürgernähe von Abgeordneten begnügen will), ist es wichtig, ihre Funktionen näher zu bestimmen. Diese Bestimmung ist nicht aus dem Begriff zu gewinnen: Nicht nur hat sich das Konzept der Zivilgesellschaft als historisch äußerst variabel gezeigt; auch seine neuzeitliche Entfaltung folgt in verschiedenen Ländern und Kulturen einer je eigenen politischen Semantik ${ }^{8}$.

\footnotetext{
$8_{\text {vgl. Koselleck } 1991}$
} 
Im Blick auf Erfordernisse wie die genannten soll versucht werden, generelle Bestimmungen der Zivilgesellschaft auszumachen.

3.

Die Zivilgesellschaft ist eine Sphäre sozialer Institutionen und Organisationen, die nicht direkt der Funktion politischer Selbstverwaltung integriert sind und nicht unmittelbar staatlicher Regulierung unterliegen, doch in verschiedener Weise auf den Staat einwirken: für ihn Grundlagen bereitstellen, Rahmenbedingungen setzen, seine Leistungen ergänzen, ihn aktiv beeinflussen. In Wirtschaft, Kultur, Bildung, Medien, Verbänden usw. erfüllt die Zivilgesellschaft Funktionen, die sich nicht in der Koordinierung von Privatinteressen erschöpfen, sondern die Konstitution eines Allgemeinen tragen. Sie bildet kollektive Identität(en), begründet Gemeinsinn, stiftet Öffentlichkeit, fördert soziale Sicherheit.

a) Die Bildung eines kollektiven Selbstbewußtseins vollzieht sich im Medium kognitiver und normativer Reflexion zugleich ${ }^{9}$. Zwar gilt in gewisser Hinsicht gerade der Staat als letztbestimmend für kollektive Identität, hinsichtlich des internen Zusammenhalts wie der Abgrenzung nach außen: Er verfügt über die stärkste zentrale Steuerungsgewalt, und er bezeichnet die härteste Grenze, die entschiedenste (notfalls im Krieg erprobte) Zugehörigkeit. In der konkreten Realität indes überlagern sich Zugehörigkeiten verschiedenster (ethnischer, kultureller, beruflicher, religiöser, ständischer etc.) Art und bilden für den einzelnen den komplexen Raum seiner Sozialität. Sie sind alle nicht nur Weisen der faktischen Grenzziehung, sondern Modi der Selbstverständigung, der Herstellung von Selbstbildern, der Reflexion über Herkunft, Zugehörigkeit und Werte. Je nach Bereich und inhaltlicher Besetzung sind solche Identitätsformationen ganz verschieden strukturiert. Unter ihnen ist die spezifisch "zivile“" Gemeinschaft zunächst durch zwei Merkmale ausgezeichnet: Sie ist auf die Regelung des gemeinsamen Lebens hin orientiert, und sie ist territorial begrenzt. Durch das erste hebt sie sich von bloßen Interessenverbänden $\mathrm{ab}$, durch das zweite von transnationalen Einheiten wie Religion, Klasse, Bildungsstand, aber auch von der Idee des Weltbürgertums; im Normalsinn unterstellt der Bürgergedanke die Teilnahme an einer partikularen Gemeinschaft. Deren Identität verlangt weder ethnische noch kulturelle Homogenität.

b) Ebenso bedeutsam wie die Ausgrenzung ist der innere Zusammenschluß und die Ausbildung der ihn tragenden Haltung: der Zivilität, des - über Gruppensolidarität hinausgehenden - aktiven Bürgersinns, des Gemeinsinns. Hier werden Grundlagen des Politischen gelegt, die für das Funktionieren der Institution vorausgesetzt sind, doch nicht von

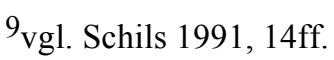


ihr erzeugt werden können. Eine zweifache Einstellung zum Allgemeinen ist erfordert: ein Interessiertsein an den gemeinsamen Angelegenheiten, das gleichzeitig ein Interesse an aktiver Beteiligung einschließt - ein eigentlich politisches Interesse -, und ein basales Interesse am Gemeinwohl, das Elemente gelebter Solidarität enthält. Bürgertugenden sind nicht in einer moralischen Umwandlung des naturgegebenen Egoismus, sondern über die Ausübung einer Praxis realisierbar, in welcher der einzene als vollberechtigtes Mitglied am Ganzen mitwirkt und zugleich für dieses Verantwortung übernimmt ${ }^{10}$. Ohne gelebtes Ethos ist kein Kollektiv überlebensfähig; keine Verfassung vermag von sich aus die motivationalen Ressourcen bereitzustellen, ohne welche sie abstrakt und tot bleibt. Das Politische - wenn dieses über staatliche Institutionen und deren Tätigkeit definiert ist - bezieht Impulse aus einem nicht- und vorpolitischen, bürgerlichen Rahmen. Das Interesse am Allgemeinen, das gelebte Vertrauen, die Teilnahme am gemeinsamen Wollen sind für jede politische Einheitsbildung und jede kollektive Selbstregulierung vorausgesetzt und durch keine noch so rational zwingenden Verträge und Argumente zu ersetzen.

c) Als drittes wirkt Zivilgesellschaft als kulturelle Öffentlichkeit. In ihr findet die Auseinandersetzung um kollektive Orientierung, um Werte und Zwecke statt, vollzieht sich der Kampf der Ideologien und der Wandel der Weltbilder. Daß diese Sphäre vom Staat freigegeben sei, ist von unmittelbarer Bedeutung für beide Seiten: Öffentlichkeit kann als staatlich regulierte nicht wirklich bestehen und sich entfalten, sondern allenfalls Sekundärfunktionen (Legitimationsbeschaffung, gesellschaftliche Selbstrepräsentanz) erfüllen; der Staat bedarf seinerseits der ihn stützenden und in Frage stellenden Öffentlichkeit. Über sie kommuniziert der einzelne mit dem Politischen, hat er an der Gestaltung der Rahmenbedingungen der Politik, an der Transformation der institutionellen Form wie der leitenden Werthaltungen und kollektiven Präferenzen teil: Ebensosehr wie in der Verstärkung partizipatorischer Mechanismen findet das monierte Demokratiedefizit seine Antwort in der Stärkung kritischer Öffentlichkeit und ihrer Einflußmöglichkeiten auf die herrschende Politik. Basisbewegungen der letzten Jahrzehnte (allen voran die ökologische) haben die Wirksamkeit einer sich verändernden öffentlichen Meinung vordemonstriert. Nach Habermas wird kommunikative Macht "im Modus der Belagerung" ausgeübt: "Sie wirkt auf Prämissen der Entscheidungsprozesse des Verwaltungssystems ohne Eroberungsabsicht ein", indem sie "den Pool von Gründen" bewirtschaftet, auf den politische Macht zur Selbstlegitimation und Selbststabilisierung angewiesen bleibt. ${ }^{11}$

d) Von der generellen Wirksamkeit der kulturellen Öffentlichkeit unterscheiden sich jene Aktivitäten, die gezielt, doch außerhalb der vom System vorgesehenen Kanäle, Einfluß auf

\footnotetext{
${ }^{10}$ Schils 1991, 48f.

${ }^{11}$ Habermas 1989, 475
} 
politische Entscheidungen nehmen. Aktive Gesetzesverletzungen ("ziviler Ungehorsam") wollen eine klar als falsch oder ungerecht wahrgenommene Politik zur Änderung nötigen; Bürgerinitiativen wollen in sachlich begrenzten Bereichen staatliche Fehlleistungen abwehren oder korrigieren, das Unvermögen von Parteien und Verwaltungen (zur Integration der Bürger, Durchsetzung von Zielen, Einsicht in neue Sachlagen) bloßlegen und kompensieren, von offiziellen Instanzen nicht wahrgenommene Funktionen erfüllen. Operiert ziviler Ungehorsam im Normalfall mit hohen moralischen Ansprüchen, so bewegen sich Bürgerinitiativen im Gesamtbereich zwischen Moral und Gruppenegoismus; beide Male sind der Staat und seine Instanzen direkter Adressat von Forderungen.

e) Nicht in dieser Weise auf den Staat gerichtet sind Formen kollektiver Selbstorganisation, die Funktionen der sozialen Sicherung, der Lebensgestaltung, der Integration erfüllen und dabei auch Elemente plebiszitärer Selbstbestimmung realisieren (Kooperativen im Arbeits-, Kultur-, Wohnbereich etc.). Teils werden Funktionslücken des Sozialstaats geschlossen; teils werden bewußt im staatsfreien Raum Sphären des Zusammenlebens eingerichtet. Doch gehören auch sie, obwohl sie sich ggf. gegen den Staat bzw. die herrschende Politik wenden, zur lebensweltlichen Grundlage des Politischen. -

Unter all diesen Hinsichten stellt die Zivilgesellschaft ein Verbindungs- und Vermittlungsglied zwischen einzelnen und dem sozialen Ganzen dar, das für die eigentlich politische Partizipation mitkonstitutiv ist. Der Zusammenschluß der atomisierten Individuen zum staatlichen Verband findet nicht unmittelbar statt, sondern über intermediäre Stadien der Einheitsbildung und gegenseitigen Abstimmung; Zivilgesellschaft ist der Titel für die an der Öffentlichkeit orientierten Typen solcher Koordinierung. Diese bilden ein Fundament staatlicher Existenz, für die sie sowohl legitimatorische wie motivationale Ressourcen abgeben; und sie üben auf das System Druck aus, beeinflussen es faktisch oder gezielt, bringen in ihm Ansprüche von einzelnen und Gruppen zum Tragen.

Fragt man sich, inwieweit diese Verhältnisbestimmung Möglichkeiten bietet, den in (2.) angeführten Desideraten gerecht zu werden, so ist keine einheitlich-globale Antwort möglich. Klar ist, daß das streng rousseauistische Postulat radikaler Demokratie nicht als solches eingelöst ist. Kollektive Souveränität kann die Autonomie des einzelnen nur vermittelt zur Geltung bringen; Institutionen der Zivilgesellschaft sind - neben analogen Vorkehrungen im Politischen Versuche, jenem Spannungsverhältnis praktikable Vermittlungsformen zu bieten. Ein Hauptgewicht besteht in der Lebendigkeit politischer Kultur. Dazu reicht nicht die Gewährleistung ihrer Minimalbedingung, der Freiheit und Liberalität, wie sie unter totalitären Regimes unter dem Titel der Zivilgesellschaft eingeklagt wird. Wesentlich ist, daß Öffentlichkeit real allgemein sei, d.h. daß sie von möglichst vielen, nicht nur von stellvertretenden 
Protagonisten getragen und wahrgenommen werde. Wenn das "vielstimmig diskutierende ... Staatsbürgerpublikum" den Platz des Souveräns einnehmen soll ${ }^{12}$, ist mehr gefordert als daß intellektuelle, kulturelle und politische Leitfiguren im offenen Disput Alternativen (in Sachfragen, normativen Orientierungen, Weltbildern) austragen; verlangt ist die möglichst breite Streuung und Verankerung des Diskurses selber in Kultur- und Bildungsinstitutionen, Medien, politischen Organisationen und sozialen Bewegungen.

4.

Hier wird deutlich, daß solche Basisnähe unmittelbar mit der institutionellen Verfaßtheit des politischen Systems verknüpft ist. Es besteht gleichsam ein fließender Übergang zwischen 'bürgerlichen' und im engen Sinn 'politischen' Tätigkeiten, Lebensbereichen und Institutionen. Gerade das Ineinander-Verschränktsein hat sein eigenes Gewicht: Es widerlegt das Scheinbild einer vorpolitischen Freiheit und Sozialität, das die civil society im Gegensatz zum Staat definiert; und es widerspricht den Kritikern einer 'korporatistischen' Politik, die den Einfluß des Staats minimalisieren möchten. In diesem Sinn hält Ch.Taylor die auf Locke und Montesquieu zurückgreifende Version der civil society jener anderen überlegen, die nur den ersten Strang aufnimmt ${ }^{13}$. Im weiteren aber macht diese Verschränkung auch die Frage interessant, welche Vorkehrungen im staatlich-institutionellen Bereich jenen Anliegen entgegenkommen, die unter dem Titel der Zivilgesellschaft artikuliert worden sind. Dazu läßt sich an Konkretisierungen auf verschiedenster Ebene denken. Nur zwei Kristallisationspunkte seien genannt: Föderalismus und direkte Demokratie.

a) Die Dezentralisierung von Meinungsbildungs- und Entscheidungsprozessen bedeutet eine Erhöhung von Betroffenheit, Kenntnisstand, kommunikativer Dichte, Eigenkompetenz, Einflußmöglichkeit, Transparenz der Entscheidungsprozeduren. Auf der Hand liegt die Analogie zur postulierten Präsenz kultureller Öffentlichkeit, ebenso die Nähe zur Kleingliedrigkeit von Gruppierungen, sozialen Bewegungen, Lebensräumen. Die für das Ganze geforderte Vermittlung von Allgemeinheit und Besonderheit gewinnt stärkere Plausibilität in überschaubaren Zusammenhängen. Das bürgerliche Leben wird nicht schon durch formale staatsbürgerliche Rechte konstituiert, sondern ereignet sich "im wirklichen Leben von wirklichen Menschen"; lokale Selbstverwaltung ist dafür ein Ort und eine Basis ${ }^{14}$. Wichtig ist, sich klarzumachen, daß die Verankerung der Öffentlichkeit im Besonderen keine Beschränkung aufs Besondere meint: Auch wenn partikulare Inhalte in der Nahperspektive auf mehr

${ }^{12}$ Habermas 1989, 468 (mit Bezug auf J. Froebel [1848])

${ }^{13}$ Ch.Taylor 1991, $79 f$.

${ }^{14}$ Dahrendorf 1991, 259f. 
Verständnis, Kompetenz und Engagement stoßen, besteht Durchlässigkeit für umfassende Zusammenhänge und globale Entscheidungen. Regionalismus und Kosmopolitismus müssen nicht Gegensätze sein; kulturelle und ethnische Pluralität wird an der Basis, in Schulen, Vereinen, Gemeinden zum Teil einsichtiger und effektvoller praktiziert als auf Staatsebene. Der Nationalstaat, ohne damit abgeschafft zu sein, verliert seine Geschlossenheit und Prädominanz gewissermaßen nach oben und unten zugleich, er gibt etwas von seiner Zentrierungs- und Steuerungsfunktion an äußere und innere Instanzen ab. Es scheint wenig sinnvoll, darüber zu spekulieren, auf welcher Ebene soziale Einheit ihre wahre Organisationsform besitzt: Die Verlegenheit der geschichtsphilosophischen Optionen - etwa die von Kant bis Toynbee debattierte Entscheidung zwischen Weltstaat und Völkerbund - ist auch heute keiner einhelligen Zielvorgabe gewichen, hat sich teils eher verschärft: Das Bewußtsein der unausweichlichen Verlagerung der Politik auf Weltebene verbindet sich mit dem Bewußtsein der Bedrohung, die darin für das Besondere und Vielfältige liegt. Immerhin haben der Gang der Dinge und die Entwicklung der Ideen die zweifache Ausrichtung akzentuiert: die Menschheitsorientierung (nicht nur als ökonomische oder militärische Zwangsläufigkeit, sondern als bejahter Horizont politischen Handelns, nicht in abstrakter Universalität, sondern von historisch gewachsenen Institutionen getragen) wie den Respekt vor dem Vielfältig-Verschiedenen. Diese doppelte Öffnung gleichzeitig zu realisieren, ist Aufgabe politischer Kultur und verlangt eine entsprechende Disponierung der Institution. Dazu ist die Diversifizierung der Ebenen politischer Partizipation, die Aufsprengung der zentralen staatlichen Fixierung ein wichtiges Element.

b) Komplementär zur Ebene ist die Form der Partizipation von Belang. Die Differenz von direkter und repräsentativer Demokratie ist in dieser Hinsicht signifikant - auch wenn auffällt, daß sie in der neueren Literatur zur Zivilgesellschaft kaum angesprochen wird. Zwar wird die Bedeutung des plebiszitären Elements in sozialen Bewegungen und Bürgerinitiativen betont, gegebenenfalls auf Basis-Selbstbestimmung in Parteien reflektiert. Doch Vorschläge zur Übertragung analoger Element in die institutionelle Politik kommen nur vereinzelt und marginal zur Sprache. Die Vitalisierung des politischen Lebens findet im Vorbereich des Systems der Machtausübung statt. Der zuweilen erhobenen Forderung nach Verstärkung des plebiszitären Elements wird mit eigentümlich schwachen Argumenten begegnet: dem Hinweis auf die in größeren Flächenstaaten nicht gegebenen vermeintlichen Voraussetzungen (eng umgrenztes Territorium, kulturelle Homogenität etc.), dem großen Entscheidungsbedarf und der Komplexität der Sachfragen, der Manipulierbarkeit der Massen und Gefährdung von Minderheiten etc. Von vornherein ist klar, daß nur eine gemischte, teil-plebiszitäre Demokratie in Frage steht; wo die Argumente den reinen Typus unterstellen, bringen sie die Diskussion in eine Abstraktionslage, die für die Thematik ohne Interesse ist. 
Nun sollen nicht die funktionalen wie normativen Probleme der direkt-demokratischen Beteiligung überspielt werden. Für das mit Rousseau definierte Demokratie-Problem bleiben die Bedingungen unverändert. Auch wenn sich der Spielraum der Einflussnahme erweitert, bleibt das Individuum in der Sachabstimmung so machtlos wie in der Wahl von Repräsentanten: Für den einzelnen wie die Minderheit bleibt die Zumutung der Legitimität des Mehrheitswillens dieselbe. Von entscheidender Bedeutung jedoch - und nur darauf soll hier hingewiesen werden sind die Auswirkungen auf politische Kultur. Tangiert sind zum einen die Inhalte. Können soziale Bewegungen und kulturelle Öffentlichkeit großangelegte thematische oder wertmäßige Veränderungen durchsetzen, so ist die Öffentlichkeit einer direkten Demokratie (zumal wenn sie auf verschiedenen Ebenen bis zur Gemeinde praktiziert wird) darüber hinaus mit konzisen Sachfragen und Gesetzesregelungen befaßt; dennoch hat sie ihren Fokus nicht (wie zuweilen unterstellt) in kommunalen oder regionalen Angelegenheiten, sondern kann sie ebensogut allgemeinste Entscheidungen betreffen. Die jeweilige Verfassung hat die diffizile Konkretisierung darüber vorzunehmen, zu welchen Themen und Vorlagen, in welcher Form und unter welchen Bedingungen Volksabstimmungen zu erfolgen haben bzw. gefordert werden können. Wichtiger als die Inhalte ist die Differenz der Form. Eine ganz andere Art von Mobilisierung der Öffentlichkeit findet statt, wenn nicht für Parteien (und deren immer komplexe und zugleich diffuse Programme) geworben wird, sondern mit Bezug auf je spezifische Sachfragen argumentiert, disputiert und entschieden wird. Ohne sich Illusionen über Sachkompetenz, politische Reife und politisches Interesse der zur Entscheidung aufgerufenen Bürger hinzugeben, ist die Bedeutung der Aufforderung zur eigenen Stellungnahme und ihre Auswirkung auf politische Reflexion von hohem Rang. Damit wird weder den Parteien ihre Funktion als organisatorischer Kern und Impulsgeber politischen Handelns noch dem Repräsentationssystem seine Aufgabe entzogen; doch stellen sie für den einzelnen in anderer Weise Vemittlungsmedien des Politischen dar, wenn nicht sie als einzige Entscheidungsträger fungieren. Gerade im Blick auf den (von der Ethik seit je betonten) Zusammenhang zwischen der Ausbildung praktischer Reflexion und eigenem Handeln ist die Differenz von Belang, ob Entscheidungen mit Bezug auf Personen (Parteien, Programme) oder Sachfragen gefällt werden: ob eine "Autorisierung" (Hobbes) anderer Personen zum Handeln oder ein eigenes Entscheiden zu Fragen des gemeinsamen Lebens stattfindet. Daß dies in einer Massendemokratie nicht fortwährend und nicht zu allem der Fall sein kann, ist unbestritten und nicht als Defizit zu beklagen. Entscheidend ist das Gegebensein der Möglichkeit, solche Stellungnahmen zu entscheidenden Fragen zu veranlassen - und natürlich ebensosehr das Ausmaß, in welchem solche Möglichkeiten in der kulturellen Öffentlichkeit verarbeitet und tatsächlich genutzt werden.

5. 
Nun ist unverkennbar, daß auch institutionelle Vorgaben wie die genannten (und andere) die Erfüllung der zivilgesellschaftlichen Desiderate nicht sichern. Beide Seiten sind nicht notwendig miteinander gekoppelt: Jede institutionelle Form kann abstrakt und ohne Leben sein. In der Realität dominiert die Klage über Demokratiedefizite wie über die mangelnde Ausübung bürgerlicher Rechte und Pflichten. Dies wirft die Frage nach Gründen solcher Defizite auf, vielleicht auch nach Grenzen der Ideale. Zum Teil wird auf Faktoren verwiesen, die mit der Struktur der modernen Gesellschaft als solcher zu tun haben. Die zunehmende Komplexität der gesellschaftlichen und sachlichen Zusammenhänge bedingt eine Intransparenz, teils Ineffizienz kollektiven Handelns, die das Engagement und Interesse an Selbstbestimmung aushöhlt. In Erfahrungen der Ohnmacht, der Auflösung tragender sozialer Gemeinsamkeiten scheint sich die von Marx unter ganz anderen Bedingungen diagnostizierte Entfremdung vom Gemeinwesen zu reproduzieren.

Indessen sind solche Hinweise vielleicht nur Teilantworten, nicht nur sofern sie ergänzungsbedürftig sind, sondern in dem radikaleren Sinn, daß das unter dem Titel einer Zivilgesellschaft umrissene Postulat unter heutigen Bedingungen überhaupt nicht adäquat zu realisieren ist. Wenn Erwartungen nicht zu erfüllen sind, legt sich ihre Revision nahe: Ähnlich wie Hegel das griechische Polis-Ideal für unwiderruflich verloren hielt, ist nach Luhmann die alteuropäische Zentrierung auf Politik unter Bedingungen der funktionalen Differenzierung nicht wiederherzustellen. Wenn jene Art von integraler Demokratie, die Rousseau vor Augen stand, sich als nicht nur unrealisierbares, sondern scheinbares Ideal herausstellt, so drängt sich in der Verlängerung dieser Einsicht die Frage auf, ob nicht ein allgemeines Abrücken von jener Zielvorstellung gefordert ist, die eine rationale Gestaltung des Gemeinwesens und kollektive Selbststeuerung (womöglich verschärft durch die Aufgabe eines vernünftigen Naturverhältnisses) mit einer maximalen Sicherung und Realisierung individueller Freiheit verknüpfen will. Es wäre eine Preisgabe analog jener, die (zumal im theoretischen Disput) mit Bezug auf klassische geschichtsphilosophische Ideale längst stattgefunden hat. Wie die PolisSittlichkeit bezeichnet möglicherweise die vollendete Demokratie eher ein bestimmtes, historisch situierbares Regulativ.

Die Preisgabe des kompakten Ideals einer gleichen Teilnahme aller an der kollektiven Selbstgesetzgebung kann zwei verschiedenen Stoßrichtungen folgen. Habermas beleuchtet die institutionelle Brechung und kulturell-öffentliche Vermittlung jener Einheit: Nach ihm geht es darum, wie "die moralische Substanz der Selbstgesetzgebung... über viele Stufen des prozeduralisierten Meinungs- und Willensbildungsprozesses auseinandergezogen" wird. ${ }^{15}$ Ideell

${ }^{15}$ Habermas 1989, 475 
bleibt solche Vermittlung auf einen einheitlichen Willen zurückbezogen. Nach einer anderen Richtung wird die funktionale Differenzierung in eine interne Pluralisierung des Allgemeinwillens übersetzt und darin ein neuer Freiheitsraum von Souveränität gesehen: Betätigung von Freiheit und Verwirklichung der volonté générale finden dann weder in kollektiver Selbstgesetzgebung noch in prozeduraler Vermittlung statt, sondern als Teilhabe an den vielfältigen und nicht systematisch koordinierbaren Entscheidungs- und Meinungsbildungsmechanismen. ${ }^{16}$ Doch auch wenn sich das radikaldemokratische Ideal, das den zivilgesellschaftichen Diskurs mitinspiriert ${ }^{17}$, als in sich problematisierbar erweist, bleibt es als Leitwert in Geltung, der unsere Frage nach Korrektiven zur bestehenden Realität mitanleitet. Die Wahrnehmung funktionaler und normativer Defizite, die im ganzen ein Fremdwerden des Politischen anzeigen, hat einen unleugbaren Brennpunkt in der 'demokratischen Frage'. Deren Beantwortung ist nicht formal und nicht im alleinigen Bezug auf die politische Institution zu geben, sondern weist auf die konkrete und vielschichtige Gestaltung des 'bürgerlichen' Lebens.

\section{Literatur}

Arato, Andrew, "Revolution, Civil Society und Demokratie", in: : Michalski, Krzysztof (Hrsg.), Osteuropa - Übergänge zur Demokratie (Transit 1/1990), S. 110-126 (Diskussionsbeiträge 127ff.) Frankfurt/M.

Ash, T.G., Ein Jahrhundert wird abgewählt, München/Wien 1990

Dahrendorf, Ralf, "Die gefährdete Civil Society", in: Michalski 1991, 247-263

Demirovic , Alex, "Zivilgesellschaft, Öffentlichkeit, Demokratie", in: Argument 185/1991, S. $41-55$

Habermas, Jürgen, "Volkssouveränität als Verfahren. Ein normatitver Begriff von Öffentlichkeit", in: Merkur 484 / 1989, S. 465-477

Honneth, Axel, "Soziologie. Eine Kolumne. Konzeptionen der "civil society"'", in: Merkur 514 / 1992, S. 61-65

Keane, John (Hrsg.), Civil Society and the State. New European Perspectives, London/New York 1988

Koselleck, Reinhart, "Drei bürgerliche Welten? Theoriegeschichtliche Vorbemerkungen zur vergleichenden Semantik der bürgerlichen Gesellschaft in Deutschland, England und Frankreich", in: Michalski 1991, 118-129

Luhmann, Niklas, Ökologische Kommunikation. Kann die moderne Gesells haft sich auf ökologische GEfahren einstellen? Opladen 1986

Michalski, Krzysztof (Hrsg.), Europa und die Civil Society. Castelgandolfo-Gespräche 1989, Stuttgart 1991

Michalski, Krzysztof (Hrsg.), Osteuropa - Übergänge zur Demokratie? (Transit - Europäische Revue; H.1), Frankfurt/M. 1990

\footnotetext{
16Demirovic 1991, 54

${ }^{17} \mathrm{Vgl}$. Arato 1990, 111
} 
Riedel, Manfred, "Der Begriff der 'bürgerlichen Gesellschaft' und das Problem seines geschichtlichen Ursprungs", in: ders., Studien zu Hegels Rechtsphilosophie, Frankfurt/M. 1969, 135-166

Riedel, Manfred, "Gesellschaft, bürgerliche", in: Geschichtliche Grundbegriffe, hrsg. von O.Brunner u.a., Stuttgart 1975, Bd.2, 719-800

Rödel,Ulrich/Frankenberg,Günter/Dubiel,Helmut, Die demokratische Frage Frankfurt/M. 1989

Schmid, Thomas, Staatsbegräbnis. Von ziviler Gesellschaft, Berlin1990

Shils, Adward, "Was ist eine Civil Society?", in: Michalski 1991, 13-51

Taylor, Charles, "Die Beschwörung der Civil Society", in: Michalski 1991, 52-81 